\title{
Diagnostic accuracy of BRCA1-associated protein 1 in malignant mesothelioma: a meta-analysis
}

\author{
Li-Ming Wang ${ }^{1}$, Zhen-Wang Shi ${ }^{1}$, Ji-Ling Wang ${ }^{2}$, Zhi Lv ${ }^{2}$, Fang-Bin Du ${ }^{2}$, Qing-Bin \\ Yang $^{2}$ and Yong Wang ${ }^{2}$ \\ ${ }^{1}$ Department of Gastroenterology, Second People's Hospital of Hefei, Anhui, China \\ ${ }^{2}$ Department of Respiratory Medicine, Second People's Hospital of Hefei, Anhui, China \\ Correspondence to: Yong Wang, email: wayt@sina.com \\ Qing-Bin Yang, email: yyangqb@126.com
}

Keywords: diagnostic accuracy, diagnostic accuracy, malignant mesothelioma, malignant mesothelioma, BAP1

Received: April 14, 2017 Accepted: August 06, $2017 \quad$ Published: August 17, 2017

Copyright: Wang et al. This is an open-access article distributed under the terms of the Creative Commons Attribution License 3.0 (CC BY

3.0), which permits unrestricted use, distribution, and reproduction in any medium, provided the original author and source are credited.

\section{ABSTRACT}

Background: Conventional measurements are not always helpful in the diagnosis of malignant mesothelioma (MM). Increasing studies indicate that loss of BRCA1associated protein 1 (BAP1) detected by immunohistochemistry (IHC) is a useful diagnostic marker for MM. In this meta-analysis, we investigated the diagnostic accuracy of BAP1 in MM.

Results: In total, 12 eligible studies with a total of 1824 patients were selected. Results indicated that loss of BAP1 sustained a pooled sensitivity of 0.56 ( $95 \%$ CI, 0.50-0.62), specificity of 1.00 (95\% CI, 0.95-1.00), PLR of 548.82 (95\% CI, 11.31-2.7 $\times 104)$, NLR of 0.44 (95\% CI, 0.39-0.50), DOR of 1247.78 (95\% CI, $25.08-6.2 \times 104)$ in discriminating MM from non-MM. The AUC of 0.72, reflecting the SROC, indicated moderate diagnostic accuracy. Subgroup analysis showed that BAP1 detection in histological specimens owned the higher diagnostic performance than cytological ones. In addition, BAP1 showed superior diagnostic accuracy in epithelioid MM than biphasic or sarcomatoid MM.

Materials and Methods: PubMed, Embase and the Cochrane Library and reference lists of related articles were searched, and studies that evaluated the utility of BAP1 in $M M$ were included. Data from eligible studies were pooled to estimate sensitivity, specificity, positive likelihood ratio (PLR), negative likelihood ratio (NLR), diagnostic odds ratio (DOR). Summary receiver operating curves (SROC) was applied to estimate overall diagnostic accuracy.

Conclusions: Current meta-analysis indicates that detection of BAP1 by IHC is a useful diagnostic marker for MM. Loss of BAP1 almost provides confirming diagnosis for MM, while positive staining for BAP1 is not enough to exclude non-MM.

\section{INTRODUCTION}

Malignant mesothelioma (MM), primarily linked to asbestos exposure, is a highly aggressive cancer that metastasizes easily, and MM patients usually have poor prognoses $[1,2]$. MM originates from the mesothelial lining cells of serosal membranes, including the pleural, peritoneum, pericardium, and tunica albuginea [3]. MM is more common in the pleura of elderly men in the western world, especially in Australia and Europe, in which patients suffering from this typically present with unexplained pleural effusion and chest pain, accompanied by weight loss and fatigue [4]. Although different therapeutic options for MM exist, there are few available effective options and its prognosis remains poor [5-7]. The likelihood of survival one year beyond 
diagnosis is $<50$ percent; thus, it is critical to accurately detect MM in early stages [1]. However, the diagnosis of $\mathrm{MM}$ is still difficult, and the challenging problem is distinguishing $\mathrm{MM}$ from benign mesothelial proliferation or adenocarcinoma on slides stained with haematoxylin and eosin. Imunohistochemistry (IHC) can provide a definitive MM diagnosis [8]. Several markers (such as p16) have been recommended in order to support the MM diagnosis [9]. However, very specific and sensitive markers for mesothelioma are absent [10].

The BRCA1-associated protein 1 (BAPl) gene, located on chromosome $3 \mathrm{p} 21.1$, is a tumour suppressor gene, and germline BAP1 mutations have been found in association with hereditary cancer syndrome. Loss of $B A P 1$ is frequently observed in $\mathrm{MM}$ and has been proven to increase the risk of MM [11-14]. BAP1 protein is a deubiquitinating enzyme, which serves as a tumour suppressor via regulation of DNA damage repair, cell cycle, and cellular differentiation. In recent years, the loss of the BAP1 protein, detected by IHC, has been widely studied for diagnosing MM. However, the results are varying, and the role of BAP1 detection in MM is still controversial. To establish whether BAP1 detection could serve as a useful tool in the diagnosis of MM, we performed a systematic review and metaanalysis by pooling relevant published studies. In addition, we assessed the diagnostic performance of BAP1 and MM subtypes on cytological or histological specimens.

\section{RESULTS}

\section{Study characteristics}

After considering independent reviews, 12 studies were included in the meta-analysis [15-26]. A flow chart, which included the reasons for study exclusion, is shown in Figure 1. Five-hundred fifty-six publications, of which 405 were duplicate studies, were obtained from the database search. After screening titles and abstracts, 128 studies were excluded for their contents that were not relevant to our study leaving 23 eligible articles. Of these, three publications were excluded because they were reviews, and six were excluded because the sensitivity or specificity calculations could not be allowed. Also, since two studies $[27,28]$ reported patient overlap with two other studies [22, 24], we chose studies containing larger subject samples or with the best quality to avoid the duplication.

Finally, 12 eligible studies with a total of 1824 patients were enrolled for the analysis. The characteristics of the eligible studies are presented in Table 1. The selected studies were published between 2015 and 2017 and included 1016 patients with confirmed MM and 808 patients with non-MM. The non-MM included non-small cell lung cancer, reactive mesothelial hyperplasia, benign mesothelioma, fibrous pleurisy, and others. All 12 studies were retrospective in design. Briefly, the types of sample are histological or/and cytological. There were two studies $[16,23]$ of which cytological and histological data reported an MM overlap. Although the data between the two types were almost the same in two studies, we chose histologial data for the reason that cytology cannot always provide a definitive diagnosis [1]. Most publications did not set cut off values for the loss of BAP1 staining while the loss was defined as nuclear staining of $<19.4 \%$ mesothelial cells in the study by Hida [22]; Shinozaki-Ushiku [25] set nuclear staining as $<10.0 \%$ mesothelial cells, and the study by Walts [26] defined absence of nuclear staining in $>50 \%$ atypical cells.

\section{Quality assessment}

The assessment by Quality assessment of diagnostic accuracy studies-2 (QUADAS-2) was summarized in Table 2. Twelve and four studies were judged to show an unclear risk of patient selection and index test, respectively, and three studies generated a high risk of bias in flow and timing. The QUADAS in the other studies displayed high quality.

\section{Diagnostic accuracy}

Figure 2 showed that the sensitivity and specificity of BAP1 staining in MM diagnosis were $0.56(95 \% \mathrm{CI}$, $0.50-0.62)$ and 1.00 (95\% CI, 0.95-1.00), respectively. The specificity in 10 studies was 1.00 , and the sensitivity in most studies was around 0.57 . Only one study showed relatively low sensitivity $(0.27)$. We also noted that pooled positive likelihood ratio (PLR) was 548.82 (95\% CI, 11.31-2.7 $\left.\times 10^{4}\right)$, pooled negative likelihood ratio (NLR) was 0.44 (95\% CI, 0.39-0.50), and pooled diagnostic OR (DOR) was $1247.78\left(95 \% \mathrm{CI}, 25.08-6.2 \times 10^{4}\right)$, while the $Q$ test $p$ value $=0.00$ and $I^{2}=87.00 \%(95 \% \mathrm{CI}$, 73.38-100.00) showed a high heterogeneity for diagnostic accuracy (Table 3).

In the summary receiver operating characteristic (SROC) graph, each point represents sensitivity against specificity in a single study, exhibiting test performance summary and trade-off between sensitivity and specificity [29]. As shown in Figure 2, the area under curve (AUC) value was $0.72(95 \% \mathrm{CI}, 0.68-0.76)$, indicating moderate diagnostic accuracy.

\section{Analysis of publication bias}

A funnel plot and Egger's test were applied to assess publication bias. The funnel plot illustrated that the point distribution was asymmetric (Figure 2), which was further confirmed by the Egger's test $(p=0.001)$. These results showed that publication bias existed. 


\section{Subgroup analysis}

Ten studies [15-18, 20-25] and five studies [16, 19, $20,23,26]$ provided data for evaluating the diagnostic accuracy of BAP1 in histological and cytological specimens, respectively. For the cytological specimens, there were no significant differences in the sensitivity or specificity compared with histological specimens (Table 3 ). However, the values of the DOR and AUC showed BAP1 detection in histological specimens demonstrated better discriminating capability than cytological ones.

Malignant mesothelioma is classified into three subtypes: 1.) epithelioid malignant mesothelioma (EMM); 2.) biphasic malignant mesothelioma (BMM); and 3.) sarcomatoid malignant mesothelioma (SMM). We also calculated the diagnostic accuracy of BAP1 detection in these three subtypes (Table 4). Five studies $[15,20$, $21,24,25]$ provided the data for the three subtypes. For EMM, the values of sensitivity, specificity, DOR, and AUC were 0.74 (95\% CI, 0.66-0.80), 1.00 (95\% CI, $0.53-1.00), 2293.52\left(95 \%\right.$ CI, 2.93-1.8 $\left.\times 10^{6}\right)$, and 0.79 (95\% CI, 0.75-0.82), respectively. For BMM, the values of sensitivity, specificity, DOR, and AUC were 0.50 (95\% CI, 0.38-0.62), 1.00 (95\% CI, 0.45-1.00), $726.23\left(95 \% \mathrm{CI}, 0.83-6.4 \times 10^{5}\right)$, and $0.58(95 \% \mathrm{CI}$, 0.54-0.62), respectively. For SMM, the values of sensitivity, specificity, DOR, and AUC were 0.07 (95\% CI, 0.00-0.72), 1.00 (95\% CI, 0.49-1.00), 52.52 (95\% CI, $\left.0.03-8.4 \times 10^{4}\right)$, and $0.86(95 \%$ CI, $0.83-0.89)$, respectively.

\section{DISCUSSION}

In this systematic review and meta-analysis, we achieved a comprehensive summary of the recently published studies investigating BAP1 diagnostic performance in MM diagnosis. We observed a fair diagnostic accuracy of BAP1 in diagnosing MM. The specificity of BAP1 detection was much higher than its sensitivity, and a good specificity-associated AUC was produced. However, the pooled estimates in present study must be interpreted with caution attributed to high heterogeneity and bias.

$\mathrm{MM}$ is a highly lethal neoplasm that is resistant to conventional treatments [30], while special treatments are not generally required in the most benign mesothelial processes, but follow-ups and patient reassurance are

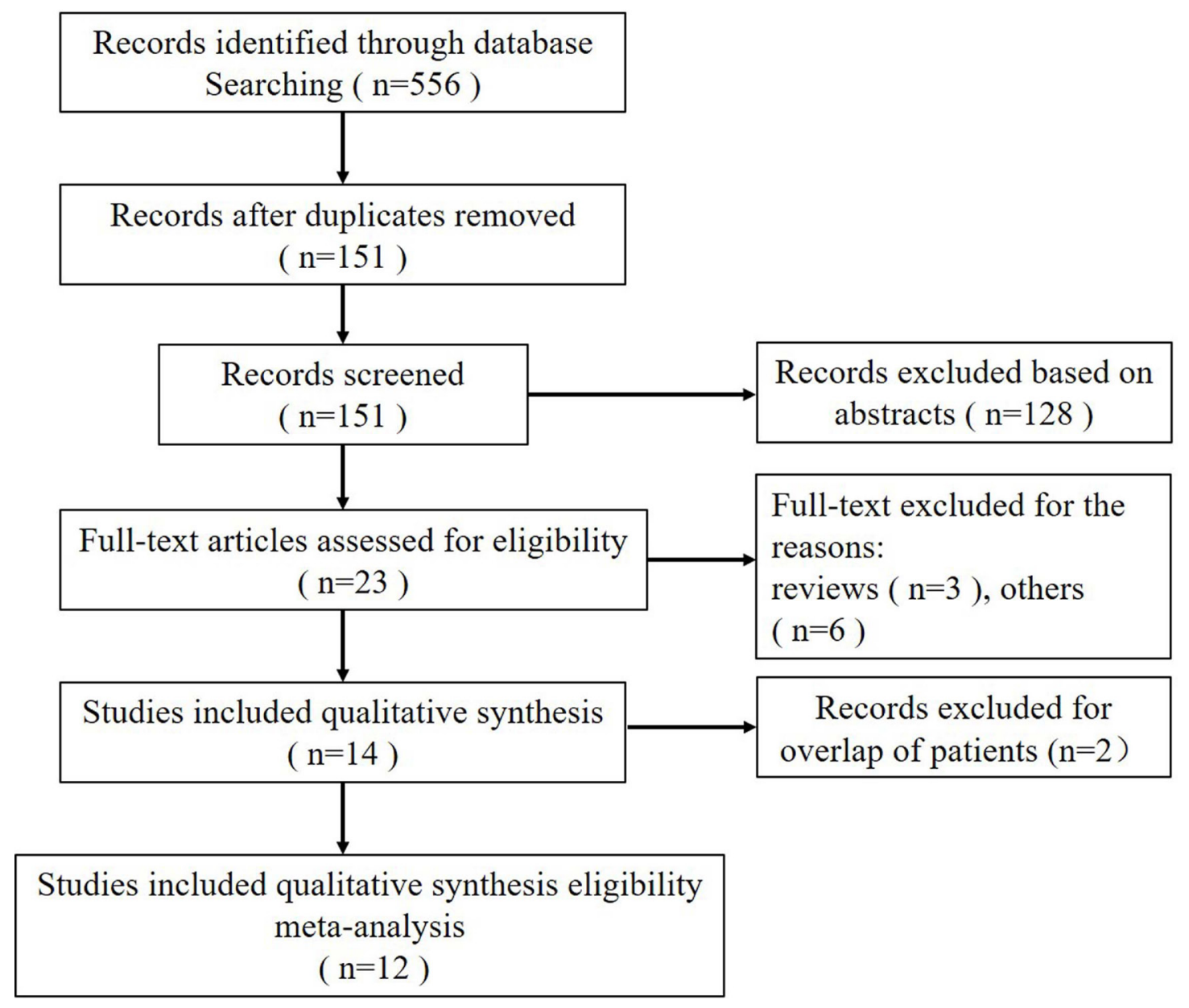

Figure 1: Flow diagram showing inclusion and exclusion of studies. 
Table 1: Characteristics of the included studies

\begin{tabular}{|c|c|c|c|c|c|}
\hline Study & Study type & Sample Type & Diagnosis/Site & $\begin{array}{c}\text { Sample size } \\
\text { MM/Non-MM }\end{array}$ & Antibody \\
\hline Andrici 2015 & Retrospective & Cytology & Pleural & $75 / 47$ & Clone C-4, Santa Cruz \\
\hline Cigognetti 2015 & Retrospective & $\begin{array}{l}\text { Histology } \\
\text { Cytology }\end{array}$ & $\begin{array}{l}\text { Pleural and } \\
\text { peritoneum }\end{array}$ & $\begin{array}{c}218 / 48 \\
45 / 17\end{array}$ & Clone C-4, Santa Cruz \\
\hline Sheffield 2015 & Retrospective & Histology & $\begin{array}{l}\text { Pleural, peritoneum } \\
\text { and pericardial } \\
\text { membrane }\end{array}$ & $31 / 52$ & Clone C-4, Santa Cruz \\
\hline McGregor 2015 & Retrospective & Histology & Pleural & $111 / 20$ & Clone C-4, Santa Cruz \\
\hline Carbone 2016 & Retrospective & Histology & Pleural & $35 / 45$ & Clone C-4, Santa Cruz \\
\hline Walts 2016 & Retrospective & Cytology & Pleural or peritoneum & $32 / 35$ & Clone C-4, Santa Cruz \\
\hline Andrici 2016 & Retrospective & Histology & Pleural or peritoneum & $286 / 395$ & Clone C-4, Santa Cruz \\
\hline Hwang 2016 & Retrospective & $\begin{array}{l}\text { Histology } \\
\text { Cytology }\end{array}$ & Pleural or peritoneum & $\begin{array}{l}15 / 3 \\
15 / 3\end{array}$ & Clone C-4, Santa Cruz \\
\hline Jaouen 2016 & Retrospective & $\begin{array}{l}\text { Histology } \\
\text { Cytology }\end{array}$ & Pleural & $\begin{array}{l}25 / 21 \\
23 / 18\end{array}$ & Clone C-4, Santa Cruz \\
\hline $\begin{array}{l}\text { Shinozaki- } \\
\text { Ushiku } 2017\end{array}$ & Retrospective & $\begin{array}{l}\text { Histology } \\
\text { Cytology }\end{array}$ & Pleural or peritoneum & $32 / 44^{*}$ & Clone C-4, Santa Cruz \\
\hline Guo 2017 & Retrospective & Histology & $\begin{array}{l}\text { Pleural or peritoneum } \\
\text { or tunica vaginalis }\end{array}$ & $22 / 35$ & Clone C-4, Santa Cruz \\
\hline Hida 2017 & Retrospective & Histology & Pleural & $51 / 25$ & Unclear, Santa Cruz \\
\hline
\end{tabular}

*Including histology and cytology.

necessary. In the meantime, many MM patients worldwide are often misdiagnosed and cannot receive proper treatment. Therefore, accurate diagnosis of mesothelioma at an early stage is a critical clinical problem. However, distinguishing MM from non-MM can be very difficult, especially with an adenocarcinoma invading the pleural cavity. MM diagnosis relies on pathology, and a definitive diagnosis often requires IHC and histochemical mucin staining [31]. BAP1 plays a role in cycle-cell progression, DNA damage repair, gene expression regulation, and chromatin remodeling. Loss of BAP1 is responsible for the process of malignant mesothelioma, and the homozygous deletion of BAPl are associated with loss of IHC staining [32], thus leading to the high specificity of BAP1 in IHC. Increasing studies have been reported for evaluating BAP1 diagnostic utility in MM. A systematic meta-analysis by Walts [26] only estimated the weighted average percentage of correct results in effusion cytology specimens and did not offer a comprehensive analysis of BAP1 in diagnosing $\mathrm{MM}$, and new, high-quality published data were also not included.

The results from our meta-analysis showed that the utility of BAP1 as a marker in the diagnosis of MM harbored a pooled sensitivity of 0.56 at a specificity of 1.00 , meaning that loss of BAP1 immunostaining almost could rule in MM, but ruling BAP1 out as a marker seems to be premature. Low BAP1 sensitivity manifested lower performance for excluding MM, and patients with positive-staining for BAP1 remain at high risk for MM. The specificity, however, was strong and could have diagnostic potential for MM in spite of the relatively low sensitivity. Of note, cells expressing one wild-type copy of BAP1 retained IHC staining, and detection of BAP1 loss may be feasible for homozygous deletion of the BAP1. However, somatic BAPl mutations have also been found and initialized in the neoplasm of sporadic MM, which does not always cause the loss of IHC staining. The BAP1 antibody (C-4, Santa Cruz) is predicted to detect wild-type BAP1 and mutant forms, and the mutant forms might not be differentiated from wild-type, thus affecting the true positive in diagnosing MM. DOR, as an independent indicator, combines sensitivity and specificity data into one measure of performance [33] with higher values indicating better discriminatory test performance. In this study, meta-analysis displayed a DOR value of 1247.78 for BAP1 detection, revealing that BAP1 could effectively 
Table 2: Details of quality assessment by the QUADAS-2

\begin{tabular}{lccccccc}
\hline \multicolumn{1}{c}{ Study } & \multicolumn{3}{c}{ Risk of bias } & \multicolumn{3}{c}{ Applicability concerns } \\
\hline Sheffield 2015 & $\begin{array}{c}\text { Patient } \\
\text { selection }\end{array}$ & Index test & $\begin{array}{c}\text { Reference } \\
\text { standard }\end{array}$ & $\begin{array}{c}\text { Flow and } \\
\text { timing }\end{array}$ & $\begin{array}{c}\text { Patient } \\
\text { selection }\end{array}$ & Index test & $\begin{array}{c}\text { Reference } \\
\text { standard }\end{array}$ \\
McGregor 2015 & $\mathrm{U}$ & $\mathrm{L}$ & $\mathrm{L}$ & $\mathrm{L}$ & $\mathrm{L}$ & $\mathrm{L}$ & $\mathrm{L}$ \\
Cigognetti 2015 & $\mathrm{U}$ & $\mathrm{L}$ & $\mathrm{L}$ & $\mathrm{L}$ & $\mathrm{L}$ & $\mathrm{L}$ & $\mathrm{L}$ \\
Andrici 2015 & $\mathrm{U}$ & $\mathrm{L}$ & $\mathrm{L}$ & $\mathrm{H}$ & $\mathrm{L}$ & $\mathrm{L}$ & $\mathrm{L}$ \\
Hwang 2016 & $\mathrm{U}$ & $\mathrm{L}$ & $\mathrm{L}$ & $\mathrm{H}$ & $\mathrm{L}$ & $\mathrm{L}$ & $\mathrm{L}$ \\
Carbone 2016 & $\mathrm{U}$ & $\mathrm{U}$ & $\mathrm{L}$ & $\mathrm{L}$ & $\mathrm{L}$ & $\mathrm{L}$ & $\mathrm{L}$ \\
Jauoen 2016 & $\mathrm{U}$ & $\mathrm{U}$ & $\mathrm{L}$ & $\mathrm{L}$ & $\mathrm{L}$ & $\mathrm{L}$ & $\mathrm{L}$ \\
Andrici 2016 & $\mathrm{U}$ & $\mathrm{L}$ & $\mathrm{L}$ & $\mathrm{L}$ & $\mathrm{L}$ & $\mathrm{L}$ & $\mathrm{L}$ \\
Walts 2016 & $\mathrm{U}$ & $\mathrm{L}$ & $\mathrm{L}$ & $\mathrm{H}$ & $\mathrm{L}$ & $\mathrm{L}$ & $\mathrm{L}$ \\
Guo 2017 & $\mathrm{U}$ & $\mathrm{L}$ & $\mathrm{L}$ & $\mathrm{L}$ & $\mathrm{L}$ & $\mathrm{L}$ & $\mathrm{L}$ \\
Shinozaki-Ushiku & $\mathrm{U}$ & $\mathrm{L}$ & $\mathrm{L}$ & $\mathrm{L}$ & $\mathrm{L}$ & $\mathrm{L}$ & $\mathrm{L}$ \\
2017 & $\mathrm{U}$ & $\mathrm{U}$ & $\mathrm{L}$ & $\mathrm{L}$ & $\mathrm{L}$ & $\mathrm{L}$ & $\mathrm{L}$ \\
Hida 2017 & $\mathrm{U}$ & $\mathrm{U}$ & $\mathrm{L}$ & $\mathrm{L}$ & $\mathrm{L}$ & $\mathrm{L}$ & $\mathrm{L}$ \\
\hline
\end{tabular}

L, low risk; U, unknown risk; $H$, high risk.

A

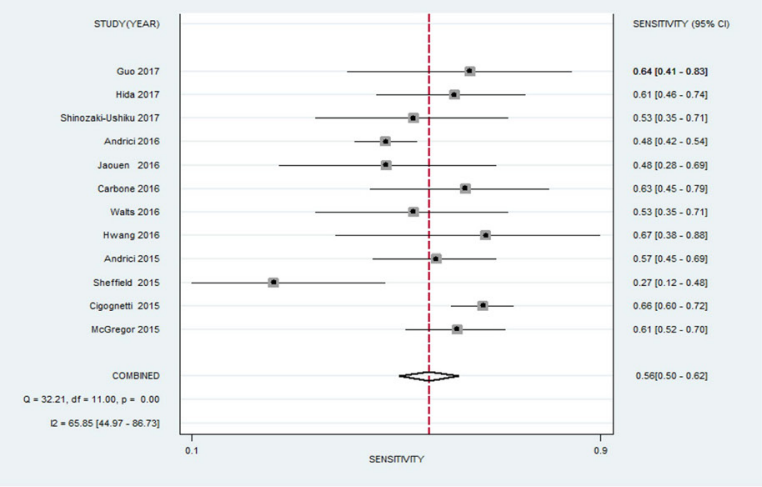

$\mathrm{C}$

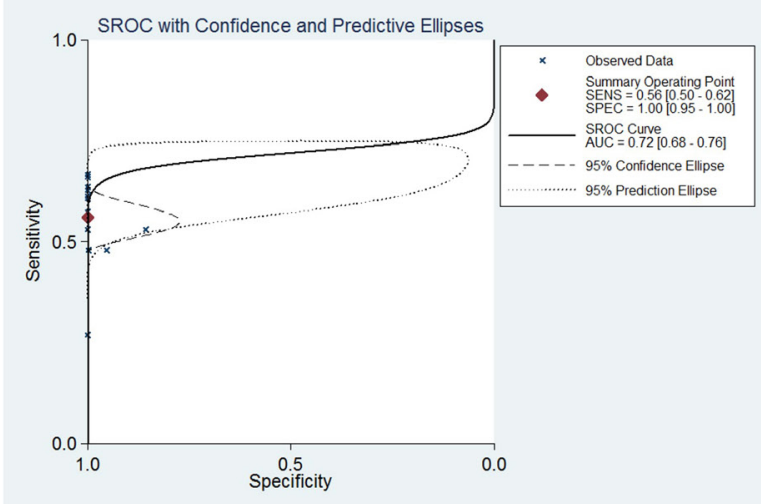

$\mathrm{B}$

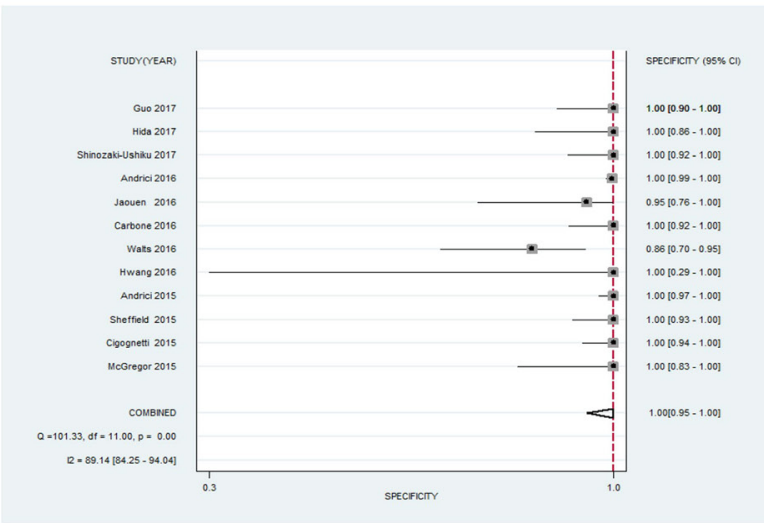

$\mathrm{D}$

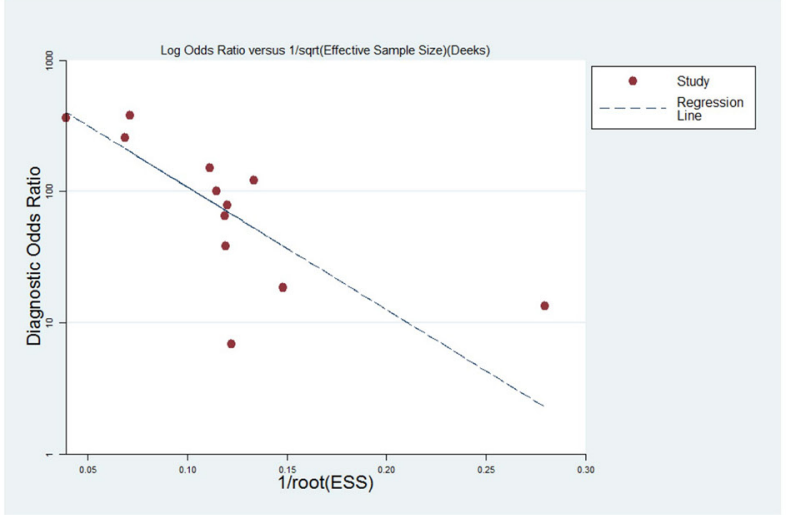

Figure 2: Forest plots for diagnostic accuracy of BRCA1-associated protein 1 (BAP1) on the diagnosis of malignant mesothelioma. Sensitivity (A), specificity (B), summary receiver operative curves (C), funnel plot (D). 
Table 3: Comparison of diagnostic accuracy of BAP1 detected in cytological specimens and histological specimens

\begin{tabular}{lcc}
\hline & Cytological specimens & Histological specimens \\
\hline Number of Studies & 5 & 10 \\
Sensitivity $(95 \%$ CI $)$ & $0.58(0.50-0.65)$ & $0.55(0.49-0.61)$ \\
Specificity $(95 \%$ CI $)$ & $0.96(0.89-0.99)$ & $1.00(0.98-1.00)$ \\
PLR $(95 \%$ CI $)$ & $20.53(8.73-62.17)$ & $180.91(35.48-6245.84)$ \\
NLR $(95 \%$ CI $)$ & $0.45(0.37-0.54)$ & $0.45(0.40-0.51)$ \\
DOR $(95 \%$ CI) & $53.47(18.42-170.91)$ & $423.81\left(63.48-1.4 \times 10^{4}\right)$ \\
AUC $(95 \%$ CI) & $0.69(0.66-0.73)$ & $0.75(0.73-0.80)$ \\
Heterogeneity & $Q$ test, $p=0.30 ; I^{2}$ statistic $=0.00$ & $Q$ test, $p=0.00 ; I^{2}$ statistic $=80.11$ \\
\hline
\end{tabular}

AUC, area under curve; DOR, diagnostic OR; NLR, negative likelihood ratio; PLR, positive likelihood ratio.

Table 4: Comparison of diagnostic accuracy of BAP1 in the epithelioid malignant mesothelioma (EMM), biphasic malignant mesothelioma (BMM), and sarcomatoid malignant mesothelioma (SMM)

\begin{tabular}{|c|c|c|c|}
\hline & EMM & BMM & SMM \\
\hline Number of Studies & 5 & 5 & 5 \\
\hline Sensitivity $(95 \% \mathrm{CI})$ & $0.74(0.66-0.80)$ & $0.50(0.38-0.62)$ & $0.07(0.00-0.72)$ \\
\hline Specificity $(95 \%$ CI) & $1.00(0.53-1.00)$ & $1.00(0.45-1.00)$ & $1.00(0.49-1.00)$ \\
\hline PLR $(95 \%$ CI $)$ & $608.72\left(0.83-4.5 \times 10^{5}\right)$ & $364.95\left(0.41-3.2 \times 10^{5}\right)$ & $49.00\left(0.03-7.0 \times 10^{4}\right)$ \\
\hline NLR $(95 \% \mathrm{CI})$ & $0.27(0.21-0.34)$ & $0.50(0.40-0.64)$ & $0.933(0.733-1.19)$ \\
\hline DOR $(95 \% \mathrm{CI})$ & $2293.52\left(2.93-1.8 \times 10^{6}\right)$ & $726.23\left(0.83-6.4 \times 10^{5}\right)$ & $52.52\left(0.03-8.4 \times 10^{4}\right)$ \\
\hline $\operatorname{AUC}(95 \% \mathrm{CI})$ & $0.79(0.75-0.82)$ & $0.58(0.54-0.62)$ & $0.86(0.83-0.89)$ \\
\hline Heterogeneity & $Q$ test, $p=0.01 ; I^{2}=75.87$ & $Q$ test, $p=0.01 ; I^{2}=76.47$ & $Q$ test, $p=0.10 ; I^{2}=36.66$ \\
\hline \multicolumn{4}{|c|}{$\begin{array}{l}\text { AUC, area under curve; DOR, diagnostic OR; NLR, negative likelihood ratio; PLR, positive likelihood ratio; EMM, } \\
\text { epithelioid malignant mesothelioma; BMM, biphasic malignant mesothelioma; SMM, sarcomatoid malignant mesothelioma. }\end{array}$} \\
\hline \multicolumn{2}{|c|}{$\begin{array}{l}\text { help discriminate MM from non-MM. Moreover, the } \\
\text { SROC curve presents a comprehensive summary of } \\
\text { diagnostic test performance, and the value of AUC for } \\
\text { BAP1 was 0.72, thus showing a moderate diagnostic } \\
\text { accuracy for MM. } \\
\text { For the DOR and SROC curve, applications in } \\
\text { clinical practice are relatively difficult, but pooling } \\
\text { likelihood ratios could provide more clinically meaningful } \\
\text { measurements [34]. The value of PLR was 548.82, }\end{array}$} & \multicolumn{2}{|c|}{$\begin{array}{l}\text { indicating that patients with MM harbored more than } \\
548.82 \text { times the possibility of BAP } 1 \text { loss as the ones } \\
\text { without MM. On the other hand, the NLR value of } 0.44 \\
\text { suggested that patients with positive BAP1 staining still } \\
\text { have a } 44 \% \text { chance of having MM; this percent is not low } \\
\text { enough to eliminate MM. Taken together, BAP } 1 \text { detection } \\
\text { by IHC could be recommended as a valid diagnostic } \\
\text { biomarker for MM, but it is not perfect. BAP1 needs to } \\
\text { be combined with other markers (such as P16) in order to }\end{array}$} \\
\hline
\end{tabular}


increase diagnostic accuracy in diagnosing MM, especially with regard to sensitivity.

Whether cytology is sufficient for a diagnosis of MM remains controversial. For this reason, we further performed a subgroup meta-analysis on the diagnostic performance of BAP1 resulting from cytological or histological specimens. We noted that the sensitivity, specificity, and NLR were nearly identical between two different specimens, but histological specimens showed superior diagnostic performance in terms of PLR, DOR, and AUC (Table 4). Meanwhile, an AUC value of 0.69 for BAP1 in cytology showed a low diagnostic accuracy for MM. Although loss of BAP1 expression in cytology could be applied to support mesothelioma diagnosis, histological specimens still have the added advantage, which is associated with the better diagnostic performance of histological diagnostic criteria when compared with cytological one.

MM has three histologic types, including EMM, BMM, and SMM, of which EMM is the most prevalent. A study reported that loss of BAP1 is rare in SMM [35]. Our data indicated that the sensitivity and DOR of BAP1 in EMM was higher than in other types. Despite an AUC value of 0.86 , a sensitivity value of 0.07 causes the utility of BAP1 for diagnosing SMM to be limited. In addition, the value of AUC in BMM was 0.58, indicating low BAP1 diagnostic accuracy. Therefore, BAP1 harbors a better diagnostic performance in diagnosing EMM when compared with the other two subtypes, and loss of BAP1 is more inclined to support an EMM diagnosis. Of note, the superior diagnostic accuracy on EMM is associated with the high frequency of EMM, which may affect the results and cause diagnostic bias.

There was higher heterogeneity in the outcomes. Meta-analyses are often accompanied with different degrees of heterogeneity, and investigations of causes for heterogeneity are important goals. The subgroup analysis data have shown that sample origins and histological types were associated with diagnostic accuracy, which may cause a high degree of heterogeneity. In addition, several studies included in present meta-analysis set the optimized cut-off values on the basis of the observations in the particular populations. Different cut-off value settings may lead to a threshold effect, thus contributing to heterogeneity. Another reason for heterogeneity may be the variance in measurement and test matrices, racial differences, and high risk of publication bias.

The strengths of our systematic review and metaanalysis were consisted of three main points: 1.) Current meta-analysis almost pooled all recently published data regarding the utility of BAP1 in diagnosing MM and provided solid evidence for the diagnostic effect of this biomarker; 2.) Subgroup analysis concluded that the detection of BAP1 in histological specimens had better diagnostic performance than cytological ones; and 3.) We also came to the conclusion that BAP1 showed superior diagnostic accuracy in EMM than BMM or SMM.
There were also potential limitations that should be taken into consideration for this analysis. First, exclusion of studies published in the form of abstracts or letters to the editor (journal) may cause publication bias. In fact, we observed obvious publication bias in current study that may distort the conclusions. This bias might derive from the fact that studies reporting a significant effect tends to be more frequently accepted for publication, while this is the reverse in studies with negative conclusions. Second, the number of included studies was small, especially in subgroup. Moreover, subjects in some studies were smallscale, which decreased the power of the studies and may overestimate the true diagnostic accuracy. Third, randomeffects modeling was used because of the significant heterogeneity in the analyses, which might have affected the results of the present study.

Despite these limitations, this meta-analysis suggested BAP1 IHC as a promising marker with extremely high specificity, and good DOR and area under curve in diagnosing malignant mesothelioma. However, BAP1 requires a combination with other biomarkers in order to improve the sensitivity.

\section{MATERIALS AND METHODS}

\section{Search strategy}

A comprehensive search was conducted including PubMed, Embase and the Cochrane Library from suitable studies to April 2017. The following keywords: ("BRCA1associated protein 1" or "BAP1") and ("mesothelin" or "mesothelioma" or "malignant mesothelioma" or "malignant mesothelin") were searched. In addition, studies were also identified by manually searching the reference of the included studies and published reviews. Studies published only in the form of abstract or letter to journal were not included for the limited data.

\section{Study selection}

We included studies that had to provide both specificity and sensitivity of BAP1 in MM diagnosis. Meanwhile, eligible studies must meet the following criteria: 1.) original studies assessing the diagnostic power of BAP1 for MM; 2.) containing true-positive, false-positive, true-negative, and false-negative data; and 3.) detection of BAP1 must be performed by IHC. Two reviewers independently evaluated the study eligibility. Disagreements were resolved through team consensus.

\section{Data extraction and quality assessment}

Data were extracted using a standard collection form. Information from each study including author name, year of publication, clinicopathological features, patient characteristics, specificity and sensitivity data, and 
methodological quality were extracted by two independent reviewers. If several studies reported the overlap of patients, we chose large-scale or the best quality study to avoid duplication. Discrepancies were resolved through team consensus.

QUADAS-2 tool $[37,38]$ was applied to assess the methodological quality of selected studies. QUADAS-2 consists of four domains including patient selection, index test, reference standard, and flow and timing. Two reviewers independently evaluated the risk of bias and concerns regarding applicability, which were rating as "low", "high" or "unclear" for one or more key domains.

\section{Statistical analyses}

The meta-analysis was performed with STATA 12.0 software (Stata Corporation; Texas, USA). The diagnostic accuracy for BAP1 loss, together with 95\% CI, was estimated by pooling sensitivity, specificity, PLR, NLR, and DOR. The sensitivity and specificity for the single test threshold identified for each study were used to plot a SROC curve [29, 39], which indicates the overall accuracy of diagnosis by AUC. AUC values of 0.5 0.7, 0.7 0.9 and $0.9 \sim 1.0$ represented low, moderate and high diagnostic accuracy, respectively. The random-effects modeling was used for meta-analysis [40].

$Q$ test and $I^{2}$ statistic were conducted to appraise heterogeneity, and $P<0.10$ was considered significant heterogeneity. $I^{2}$ values of $50 \% \sim 75 \%$ and $75 \% \sim 100 \%$ were considered to have moderate and high heterogeneity, respectively. Stata 12.0 software was used to make funnel plot and Egger test was conducted for evaluating publication bias where $P$-value $<0.05$ suggested the publication bias existed. Funnel plots and Egger test were used to assess publication bias [41].

\section{Abbreviations}

BAP1 = BRCA1-associated protein 1; $\mathrm{MM}=$ malignant mesothelioma; IHC = imunohistochemistry; AUC $=$ area under curve; $\mathrm{DOR}=$ diagnostic $\mathrm{OR} ; \mathrm{NLR}=$ negative likelihood ratio; $\mathrm{PLR}=$ positive likelihood ratio; $\mathrm{MM}=$ malignant mesothelioma; EMM = epithelioid malignant mesothelioma; $\mathrm{BMM}=$ biphasic malignant mesothelioma; $\mathrm{SMM}=$ sarcomatoid malignant mesothelioma; QUADAS-2 $=$ quality assessment of diagnostic accuracy studies-2; $\mathrm{SROC}=$ summary receiver operating characteristic.

\section{Author contributions}

L.M.W. and Y.W. takes responsibility for the integrity of this study; Y.W. and Q.-B.Y. designed and supervised the study; Y.W. and Q.-B.Y. contributed to the study design, data acquisition and interpretation; Y.W. drafted manuscript; Z-W.S., J-L.W., Z.L., F-B.D., Y.W., and Q.B.Y. analyzed data and revised manuscript. All authors approved the final manuscript.

All authors are aware of and agree to the content of the paper and their being listed as an author. There are no potential conflicts of interest exist with any companies/ organizations whose products or services may be discussed in this article.

\section{CONFLICTS OF INTEREST}

None.

\section{REFERENCES}

1. Robinson BW, Musk AW, Lake RA. Malignant mesothelioma. Lancet. 2005; 366:397-408.

2. Bonelli MA, Fumarola C, La Monica S, Alfieri R. New therapeutic strategies for malignant pleural mesothelioma. Biochem Pharacol. 2017; 123:8-18.

3. Suster S, Moran C. Malignant mesothelioma: current status of histopathologic diagnosis and molecular profile. Expert Rev Mol Diagn. 2005; 5:715-723.

4. Scott B, Mukherjee S, Lake R, Robinson B. Malignant mesothelioma. In: Hanson H, Editor. Textbook of lung cancer. London: Martin Dunitz; 2000. pp. 273-293.

5. West S, Lee Y. Management of malignant pleural mesothelioma. Clin Chest Med. 2006; 27:335-354.

6. Chapman A, Mulrennan S, Ladd B, Muers MF. Population based epidemiology and prognosis of mesothelioma in Leeds, UK. Thorax. 2008; 63:435-439.

7. Gemba K, Fujimoto N, Aoe K, Kato K, Takeshima Y, Inai K, Kishimoto T. Treatment and survival analyses of malignant mesothelioma in Japan. Acta Oncol. 2013; 52:803-808.

8. Ordóñez NG. Immunohistochemical diagnosis of epithelioid mesotheliomas: a critical review of old markers, new markers. Hum Pathol. 2002; 33:953-967.

9. Hamasaki M, Matsumoto S, Abe S, Hamatake D, Kamei T, Hiroshima K, Kawahara K, Sato A, Tsujimura T, Nakatani Y, Yoshida Y, Iwasaki A, Nabeshima K. Low homozygous/ high heterozygous deletion status by p16 FISH correlates with a better prognostic group than high homozygous deletion status in malignant pleural mesothelioma. Lung Cancer. 2016; 99:155-161.

10. Ordóñez NG. Application of immunohistochemistry in the diagnosis of epithelioid mesothelioma: a review and update. Hum Pathol. 2013; 44:1-19.

11. Testa JR, Cheung M, Pei J, Below JE, Tan Y, Sementino E, Cox NJ, Dogan AU, Pass HI, Trusa S, Hesdorffer M, Nasu M, Powers A, et al. Germline BAP1 mutations predispose to malignant mesothelioma. Nat Genet. 2011; 43:1022-1025.

12. Sekido Y. Molecular pathogenesis of malignant mesothelioma. Carcinogenesis. 2013; 34:1413-1419.

13. Carbone M, Ferris LK, Baumann F, Napolitano A, Lum CA, Flores EG, Gaudino G, Powers A, Bryant-Greenwood P, 
Krausz T, Hyjek E, Tate R, Friedberg J, et al. BAP1 cancer syndrome: malignant mesothelioma, uveal and cutaneous melanoma, and MBAITs. J Transl Med. 2012; 10:179.

14. White A, Harper J. Cancer. Emerging anatomy of the BAP1 tumor suppressor system. Science 2012; 337:1463-1464.

15. Carbone M, Shimizu D, Napolitano A, Tanji M, Pass H, Yang $\mathrm{H}$, Pastorino S. Positive nuclear BAP1 immunostaining helps differentiate non-small cell lung carcinomas from malignant mesothelioma. Oncotarget. 2016; 7:59314-59321. https://doi. org 10.18632/oncotarget.10653.

16. Hwang H, Sheffield B, Rodriguez S, Thompson K, Tse C, Gown A, Churg A. Utility of BAP1 Immunohistochemistry and p16 (CDKN2A) FISH in the Diagnosis of Malignant Mesothelioma in Effusion Cytology Specimens. Am J Surg Pathol. 2016; 40:120-126.

17. Sheffield B, Hwang H, Lee A, Thompson K, Rodriguez S, Tse C, Gown A, Churg A. BAP1 immunohistochemistry and p16 FISH to separate benign from malignant mesothelial proliferations. Am J Surg Pathol. 2015; 39:977-982.

18. Andrici J, Jung J, Sheen A, D’Urso L, Sioson L, Pickett J, Parkhill TR, Verdonk B, Wardell KL, Singh A, Clarkson A, Watson N, Toon CW, Gill AJ. Loss of BAP1 expression is very rare in peritoneal and gynecologic serous adenocarcinomas and can be useful in the differential diagnosis with abdominal mesothelioma. Hum Pathol. 2016; 51:9-15.

19. Andrici J, Sheen A, Sioson L, Wardell K, Clarkson A, Watson N, Ahadi MS, Farzin M, Toon CW, Gill AJ. Loss of expression of BAP1 is a useful adjunct, which strongly supports the diagnosis of mesothelioma in effusion cytology. Mod Pathol. 2015; 28:1360-1368.

20. Cigognetti M, Lonardi S, Fisogni S, Balzarini P, Pellegrini V, Tironi A, Bercich L, Bugatti M, Rossi G, Murer B, Barbareschi M, Giuliani S, Cavazza A, et al. BAP1 (BRCA1-associated protein 1) is a highly specific marker for differentiating mesothelioma from reactive mesothelial proliferations. Mod Pathol. 2015; 28:1043-1057.

21. Guo Z, Carbone M, Zhang X, Su D, Sun W, Lou J, Gao Z, Shao D, Chen J, Zhang G, Hu J, Chen K, Wang F, et al. Improving the Accuracy of Mesothelioma Diagnosis in China. J Thorac Oncol. 2017; 12:714-723.

22. Hida T, Hamasaki M, Matsumoto S, Sato A, Tsujimura T, Kawahara K, Iwasaki A, Okamoto T, Oda Y, Honda $\mathrm{H}$, Nabeshima K. Immunohistochemical detection of MTAP and BAP1 protein loss for mesothelioma diagnosis: Comparison with 9p21 FISH and BAP1 immunohistochemistry. Lung Cancer. 2017; 104:98-105.

23. Jaouen A, Thivolet-Bejui F, Chalabreysse L, Piaton E, Traverse-Glehen A, Isaac S, Decaussin-Petrucci M, Depaepe L, Fontaine J, Remy I, Maury JM, Brevet M. BRCA1 associated protein 1 (BAP1) expression in pleural diffuse malignant mesothelioma: A comparative cytological and histological analyses on 50 patients. Ann Pathol. 2016; 36:111-119.

24. McGregor SM, Dunning R, Hyjek E, Vigneswaran W, Husain AN, Krausz T. BAP1 facilitates diagnostic objectivity, classification, and prognostication in malignant pleural mesothelioma. Hum Pathol. 2015; 46:1670-1678.

25. Shinozaki-Ushiku A, Ushiku T, Morita S, Anraku M, Nakajima J, Fukayama M. Diagnostic utility of BAP1 and EZH2 expression in malignant mesothelioma. Histopathol. 2017; 70:722-733.

26. Walts AE, Hiroshima K, McGregor SM, Wu D, Husain AN, Marchevsky AM. BAP1 Immunostain and CDKN2A (p16) FISH Analysis: Clinical Applicability for the Diagnosis of Malignant Mesothelioma in Effusions. Diagn Cytopathol. 2016; 44:599-606.

27. Hida T, Hamasaki M, Matsumoto S, Sato A, Tsujimura T, Kawahara K, Iwasaki A, Okamoto T, Oda Y, Honda H, Nabeshima K. BAP1 immunohistochemistry and p16 FISH results in combination provide higher confidence in malignant pleural mesothelioma diagnosis: ROC analysis of the two tests. Pathol Int. 2016; 66:563-570.

28. McGregor SM, McElherne J, Minor A, Keller-Ramey J, Dunning R, Husain AN, Vigneswaran W, Fitzpatrick C, Krausz T. BAP1 immunohistochemistry has limited prognostic utility as a complement of CDKN2A (p16) fluorescence in situ hybridization in malignant pleural mesothelioma. Hum Pathol. 2017; 60:86-94.

29. Jiang J, Shi HZ, Liang QL, Qin SM, Qin XJ. Diagnostic value of interferon-gamma in tuberculous pleurisy: a meta analysis. Chest. 2007; 131:1133-1141.

30. Kumar P, Kratzke RA. Molecular prognostic markers in malignant mesothelioma. Lung Cancer. 2005; 49:S53-60.

31. Husain A, Colby T, Ordonez N, Krausz T, Attanoos R, Beasley M, Borczuk A, Butnor K, Cagle P, Chirieac L, Churg A, Dacic S, Fraire A, et al. Guidelines for pathologic diagnosis of malignant mesothelioma: 2012 update of the consensus statement from the International Mesothelioma Interest Group. Arch Pathol Lab Med. 2013; 137:647-667.

32. Churg A, Sheffield B, Galateau-Salle F. New Markers for Separating Benign From Malignant Mesothelial Proliferations: Are We There Yet? Arch Pathol Lab Med. 2016; 140:318-321.

33. Glas A, Lijmer J, Prins M, Bonsel G, Bossuyt P. The diagnostic odds ratio: a single indicator of test performance. J Clin Epidemiol. 2003; 56:1129-1135.

34. Deeks J. Systematic reviews in health care Systematic reviews of evaluations of diagnostic and screening tests. BMJ. 2001; 323:157-162.

35. Hwang H, Pyott S, Rodriguez S, Cindric A, Carr A, Michelsen C, Thompson K, Tse C, Gown A, Churg A. BAP1 Immunohistochemistry and p16 FISH in the Diagnosis of Sarcomatous and Desmoplastic Mesotheliomas. Am J Surg Pathol. 2016; 40:714-718.

36. Alakus H, Yost SE, Woo B, French R, Lin GY, Jepsen K, Frazer KA, Lowy AM, Harismendy O. BAP1 mutation is a frequent somatic event in peritoneal malignant mesothelioma. J Trans1 Med. 2015; 13:122.

37. Whiting P, Rutjes A, Reitsma J, Bossuyt P, Kleijnen J. The development of QUADAS: a tool for the quality assessment 
of studies of diagnostic accuracy included in systematic reviews. BMC Med Res Methodol. 2003; 10:25.

38. Whiting P, Rutjes A, Westwood M, Mallett S, Deeks J, Reitsma J, Leeflang M, Sterne J, Bossuyt PQ-G. QUADAS-2: A Revised Tool for the Quality Assessment of Diagnostic. Ann Intern Med. 2011; 155:529-536.

39. Moses L, Shapiro D, Littenberg B. Combining independent studies of a diagnostic test into a summary ROC curve: data- analytic approaches and some additional considerations. Stat Med. 1993; 12:1293-1316.

40. DerSimonian R, Laird L. Meta-analysis in clinical trials. Control Clin Trials. 1986; 7:177-188.

41. Egger M, Davey Smith G, Schneider M, Minder C. Bias in meta-analysis detected by a simple, graphical test. BMJ. 1997; 315:629-634. 